\title{
The Research on Automatic Detection Technology of Product Size Based on Machine Vision
}

\author{
Denghui $\mathrm{Li}^{1, \mathrm{a}}$, Yanhong Wang ${ }^{2, b}$ \\ ${ }^{1}$ Institute of Information Technology Guilin University of Electronic Technology, GUILIN, China \\ ${ }^{2}$ Guilin University of Electronic Technology, GUILIN, China \\ aldhui@guet.edu.cn, bwangyh@guet.edu.cn
}

Keywords: machine vision; automatic measurement; edge detection;

\begin{abstract}
It is important that product size can be checked quickly and accurately on the production line. The speed and precision are slowed by traditional manual measurement and the speed based on machine vision in the paper is obviously improved. First of all, the collected image is converted into the correct format and the noise is removed. The second, the image edge of product is detected. Finally, the product image segmentation and matching is done and the coordination is transformed. The test shows that the product is measured by the method in the paper quickly and accurately. It can be satisfied with the requirement of on-line.
\end{abstract}

\section{Introduction}

The traditional detection methods have not met the needs of production with the development of modern industry. A new kind of production measurement is a new technology based on machine vision. ${ }^{[1-3]}$ The new measurement technology is based on the modern optics by image technology in recent years. It compromises optoelectronics, computer vision and information processing.

The development of computer vision makes it possible to measure automatically. ${ }^{[4-5]}$ Many productions have the shape of diversity and complexity. In the paper, the image preprocessing, production position and segmentation have been done to complete the size measurement. The size is compared with standard template to determine whether a product is qualified.

\section{Product image preprocessing}

The camera is an important machine in machine vision system. ${ }^{[6-9]}$ The light signal is converted to orderly electrical signal by camera. The product image on the line is got by camera, and the image format can also be various. The JPGE format is a loss compression. The image storage is solved by JPEG compression without damage the quality of the image. Other format image is converted into JPGE format, and the next step of image preprocessing can be done.

Average filtering is the traditional de-noising method. The formula is such as (1) below:

$$
g(x, y)=\frac{1}{M} \sum_{(i, j \subseteq s)} f(i, j)
$$

Here, $f(i, j)$ is the original image matrix. $g(x, y)$ is the processed image matrix.

Median filtering is also a practical method de-noising in time domain. It can eliminate impulse noise very well. The formula is such as (2) below:

$$
y_{i}=\operatorname{Med}\left\{f_{i-v}, \cdots f_{i}, \cdots f_{i+v}\right\} \quad i \in N, v=\frac{m-1}{2}
$$

Here, the constant of $m$ is the length of the window.

The image noise is eliminated by the before two kinds of methods, and the image edge is also lost. This make the image processed become blurred. The noise can be eliminated and the edge details can be kept at the same time based on wavelet.

The noisy image can be represented as shown (3) below:

$$
g(x, y)=f(x, y)+\varepsilon(x, y)(x, y=1, \cdots N)
$$


Here, $\varepsilon(x, y)$ is the noise component. $f(x, y)$ is ideal image.

Formula (3) is transformed in wavelet domain as follow:

$Y(i, j)=X(i, j)+V(i, j) \quad(i, j=1, \cdots N)$

Here, $Y(i, j)$ is a noisy wavelet coefficient, $V(i, j)$ is a noisy wavelet coefficient.

The image is divided into a low-pass component LL and three high-pass components (HL, LH and $\mathrm{HH}$ ) in the wavelet domain. Because the useful information (LL)is mainly concentrated in the low frequency area, and the noise are in the high frequency part(HH). The noise is removed by setting the threshold. The formula is such as (5) below:

$$
W_{r}=\left\{\begin{array}{cc}
W & |W| \geq T \\
0 & |W|<T
\end{array}\right.
$$

Here, $T=\sigma \sqrt{2 \ln (N)}$ is the threshold. $W$ is the wavelet transform coefficient matrix, and $W_{r}$ is the wavelet coefficient matrix by threshold. At last, the de-noising image is got by reconstruction. The original product image is such as Fig 1 below, and the image of removed noise is shown in Fig 2.

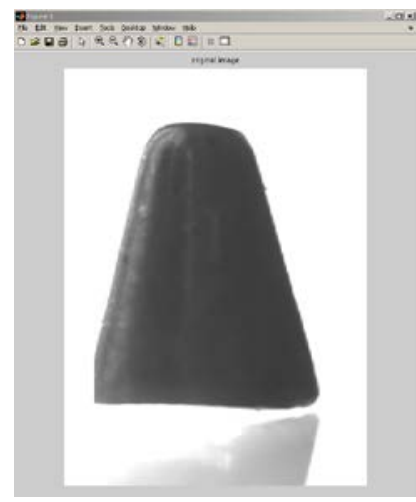

Fig1 original image

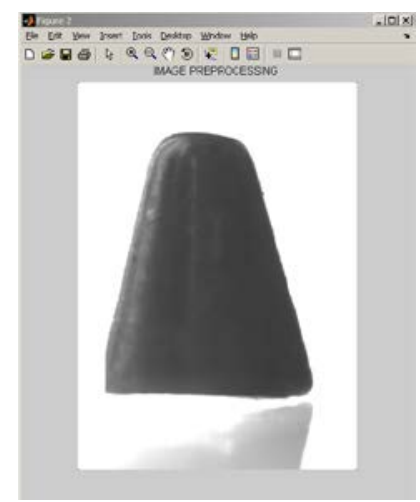

Fig2 filtered image

If the image contrast is smaller because of the light problem when the image is collected, the gray transformation should be taken to enhance the image contrast. The segmentation to enhance contrast is adopted in this paper. It is as follows:

$$
g=\left\{\begin{array}{cc}
(c / a) f & 0 \leq f<a \\
{[(d-c) /(b-a)][f-a]+c} & a \leq f<b \\
{\left[(M-d) /\left(M_{f}-b\right)\right][f-b]+d} & b \leq f<M_{f}
\end{array}\right.
$$

Here, $M_{f}$ is the highest grey value before transformation. $M_{g}$ is the maximum of grey value after transformation. The constant of a, b, c, $d$ is the different grey level.

\section{Image binarization}

Image binary is a widely technology, it uses the difference of the target and its background on the gray characteristic. The original image is turned into binary image by only two gray values. Assuming the image $f$, its grayscale is $L$ and $T$ is its threshold value. The image is divided into two areas, the pixels of gray level $0 \sim \mathrm{T}$ and $\mathrm{T}+1 \sim \mathrm{L}-1$ are belonged to area $\mathrm{A}$ and area $\mathrm{B}$. The variance formula is such as (7) below:

$$
\partial=P_{A}\left(u_{A}-\bar{u}\right)^{2}+P_{B}\left(u_{B}-\bar{u}\right)^{2}
$$

Here, $P_{A}$ and $P_{B}$ is the probability of area A and area B respectively. The variable of $u_{A}$ and $u_{B}$ is the average gray for area $\mathrm{A}$ and area $\mathrm{B}$ respectively. The variable of $\bar{u}$ is the average gray of whole image. The simulation result is shown in Fig 3. 


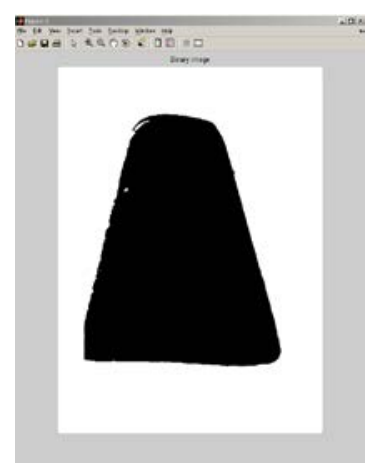

Fig3 binary image



Fig4 filled image

The border of binary image is coarser. There are some holes in the region of the object. This kind of situation can be improved by continuous opening operation and closing operation. The desired effect can be produced by several times corrosion and expansion. The image filled is as shown in Fig 4.

\section{Contour extraction}

Edge detection is the important step in the process of product size automatic measurement. It contains the useful information for product identification.

Edge detection based on mathematical morphology is adopted in this paper in order to extract image edge information and restrain noise. The formula is such as (8) below:

$$
D=(A \circ B) \oplus B-(A \cdot B) \mathrm{X} \theta B
$$

Here, $A$ is the image matrix, and $B$ is the appropriate structure element. The edge information of product image can be detected effectively by formula (8). The noise of the peak is restrained. The simulation result is shown in Fig 4.The product edge position is accurate and smooth in Fig 5, and it has the stronger ability to resist noise. The linear fitting is adopted based on the least square method. The result out of product size is as follows in Fig 6.

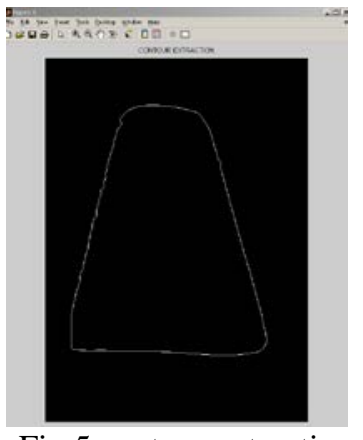

Fig 5 contour extraction

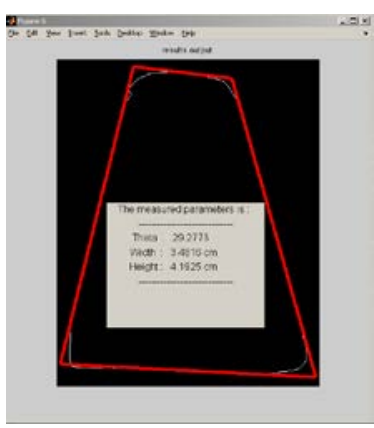

Fig 6 result out

\section{Simulation analysis}

In order to verify the effectiveness of the method, some products are experimented in this paper. The process is finished by MATLAB simulation environment. Assuming that the standard size of several products is $D_{1} \sim D_{N}$ respectively, the products are measured based on machine vision. The error is as shown in table1.

TABLE I. EDGE SIZE MEASUREMENTS

\begin{tabular}{cllll}
\hline $\begin{array}{c}\text { Product } \\
\text { name }\end{array}$ & $\begin{array}{l}\text { Binary } \\
\text { points }\end{array}$ & $\begin{array}{l}\text { Conversion } \\
\text { results(cm) }\end{array}$ & $\begin{array}{l}\text { Measurement } \\
\text { results(cm) }\end{array}$ & $\begin{array}{l}\text { Error } \\
\text { (cm) }\end{array}$ \\
\hline P1 & 200 & 5.325 & 5.319 & 0.006 \\
\hline P2 & 300 & 6.190 & 6.280 & -0.09 \\
\hline P3 & 250 & 7.055 & 7.085 & -0.03 \\
\hline P4 & 350 & 8.755 & 8.687 & 0.068 \\
\hline
\end{tabular}


The method based on binary image can be more accurate to check the shape of product by comparing the standard data from above table. The error range is allowed, and the general industrial detection can be satisfied. Therefore, the automatic detection technology based on machine vision can be used to on-line production.

\section{Conclusion}

The automatic detection of product based on image processing technology is the new and hot research topic. It has advantages of non-contact, high accuracy and fast speed compared with traditional manual measurement. This method will become a kind of important measuring technology with the development of computer and image processing.

The following issues remain will be further solved. The hardware based on special signal processing chip (DSP) is added in the measurement system. The measurement is only applied to the two-dimensional image geometry size. The three-dimensional image can be adapted to the further improvement method.

\section{References}

[1] Yan Liu,Feihong Yu. Automatic inspection system of surface defects on optical IR-CUT filter based on machine vision[J]. Optics and Lasers in Engineering . 2014

[2] Mohammad H. Karimi,Davud Asemani. Surface defect detection in tiling Industries using digital image processing methods: Analysis and evaluation[J]. ISA Transactions . 2013

[5] Zhu Hongbo,Xu Xuejun,Wang Jing,Chen Xuesong,Jiang Shaohua. A Rapid Automatic Image Registration Method Based on Improved SIFT[J]. Procedia Environmental Sciences . 2011

[6] Feng Duan,Yao-Nan Wang,Huan-Jun Liu,Yang-Guo Li. A machine vision inspector for beer bottle[J]. Engineering Applications of Artificial Intelligence . 2007 (7)

[7] Zhen Zhou,Qing Li,Xiong Li,Ren Yuan Tong,Ge Shi. An Image Measuring Method for the Fabric Defect Detection System[J]. Applied Mechanics and Materials . 2013 (333)

[8] A. Ghosh,T. Guha,R.B. Bhar,S. Das. Pattern classification of fabric defects using support vector machines[J]. International Journal of Clothing Science and Technology . 2011 (2).

[9] K.L. Mak,P. Peng,K.F.C. Yiu. Fabric defect detection using morphological filters[J]. Image and Vision Computing . 2009 (10). 